\title{
Einleitende Gedanken: Was für die Geschichte übrig bleibt
}

Colonisateurs du monde, nous voulons que tout nous parle: les bêtes, les morts, les statues. ${ }^{1}$

\section{Was wir über die Vergangenheit wissen woll(t)en}

„Was war es, was wir wissen wollten? [...] Was war es, was wir erhoffen durften?“`2

Diese Fragen stellte sich der Philosoph Hans Blumenberg 1981, exakt zwei Jahrhunderte nach dem Erscheinen von Immanuel Kants Kritik der reinen Vernunft (1781). Mit diesen Fragen verdeutlichte er die Verschiebung des erkenntnistheoretischen Interesses, die sich in der Zeitspanne zwischen dem Ende des 18. und dem des 20. Jahrhunderts vollzogen hatte. Die klassische Kant'sche Frage danach, was wir wissen können, habe sich in die Frage danach verwandelt, was wir wissen wollen und wollten. Dieser letzteren Fragestellung gelte das Interesse der Erkenntnistheorie im ausgehenden 20. Jahrhundert. Die Quellen, mit deren Hilfe Blumenberg diese Fragen zu beantworten suchte, waren die historischen Varianten von „absoluten Metaphern“ und Mythologemen. In ihrem tausendjährigen Überleben und gleichzeitig ihrer ständigen Anpassung an die jeweils gegenwärtigen Fragen und Herausforderungen gäben Mythen und Metaphern Auskunft über die (unbewussten) kollektiven Wünsche, Hoffnungen und Erwartungen der Zeitgenossen.

An dieser Stelle gilt das Interesse nicht so sehr Blumenbergs philosophischanthropologischem Entwurf, sondern vielmehr seiner erkenntnistheoretischen Frage, die sich in Bezug auf die Geschichtswissenschaft und -schreibung als ergiebig erweist, um neue Perspektiven für die Geschichte der Geschichtswissenschaft zu erschließen. Fragt man im historiografischen Kontext: „Was war es, was wir wissen wollten?“, rücken zunächst die Wünsche, Erwartungen und Orientierungsbedürfnisse der jeweiligen Gegenwart, die mit der zu rekonstruierenden Vergangenheit verbunden waren und sind, in den Mittelpunkt. Auch diese Erwartungen zählen zu den geschichtlichen Fakten, die der historischen Untersu-

1 Chris Marker: Les statues meurent aussi. In: Ders.: Commentaires. Bd.1. Paris 1961, S.7-23, hier S. 13.

2 Hans Blumenberg: Die Lesbarkeit der Welt (1981). 5. Aufl. Frankfurt a.M. 2000, S. 9. 
chung würdig sind. Ihre Erkundung gibt Aufschluss darüber, welche vergangenen Sachverhalte und Sinnzusammenhänge für die jeweilige Gegenwart von Bedeutung waren bzw. sind, welche Vorstellung von der Geschichtsschreibung, von ihrem Erkenntnispotenzial und von ihrer eigenen Orientierungsrolle Historiker/innen jeweils besaßen und besitzen, ferner darüber, welche Hoffnungen und Erwartungen sie an diese Vergangenheit hegten und immer noch hegen.

Die Frage danach, was wir wissen woll(t)en, wird dann besonders spannend, wenn das Wissen-Wollen über die Vergangenheit die erkenntnistheoretischen und methodischen Grenzen des Wissen-Könnens überschreitet. Denn im Vergleich zu dem historischen Material, das als absichtliche Hinterlassenschaft oder zumindest als unbewusstes Relikt zu uns gelangt ist, weist der Wille zum Wissen - oder in Johann Gustav Droysens Worten: die „historische Frage“3 - Überschusscharakter auf. Das überlieferte Material bestimmt mit seiner Dürftigkeit und Unvollständigkeit die Grenzen sowie die Bedingungen der historischen Erkenntnis. Dies wiederum ist der Fall, weil die Geschichte ihren Anspruch auf Wissenschaftlichkeit einerseits aufgrund der Fundierung ihrer Aussagen mit empirischen Belegen und andererseits aufgrund ihrer intersubjektiven Überprüfbarkeit erheben kann. Quellen (und Überreste) haben dabei ein Vetorecht, nicht, weil sie vorgeben, was zu sagen sei, sondern, weil sie Historiker/-innen verbieten, beliebige sowie unwahre Aussagen auszusprechen. ${ }^{4}$ Die Empfehlung an sie lautet daher: „Stelle deine vom Orientierungsbedarf der Gegenwart angetriebenen historischen Fragen so, dass sie auch forschend beantwortet werden können." ${ }^{\text {5 }}$

Dennoch - und das ist ein zentraler Aspekt des vorliegenden Sammelbandes - lässt sich im Verlauf der Geschichte der Geschichtsschreibung immer wieder feststellen, dass, abhängig davon, was man wissen wollte, stets neue Arten von in der Gegenwart bestehenden Spuren der Vergangenheit als historisches Material verwertet wurden. In der Tat hängt die Entscheidung darüber, welche gegenwärtigen „Dinge“6 als Träger vergangener Informationen für die Forschung nutzbar gemacht werden können, mit vielen Faktoren zusammen. Gegenwärtig bestehende Dinge, die ein bestimmtes Alter aufweisen, sind nicht an sich und

3 Johann Gustav Droysen: Historik. Hrsg. von Peter Leyh. Stuttgart-Bad Cannstatt 1977, passim. 4 Reinhart Koselleck: Standortbindung und Zeitlichkeit. Ein Beitrag zur historiographischen Erschließung der geschichtlichen Welt. In: Objektivität und Parteilichkeit in der Geschichtswissenschaft. Hrsg. von Reinhart Koselleck [u. a.]. München 1977, S. 17-46, hier S. 45.

5 Jörn Rüsen: Historik. Theorie der Geschichtswissenschaft. Köln [u. a.] 2013, S. 176.

6 Der Begriff „Ding“ wird hier als der neutralste verwendet, um das zu bezeichnen, „was in einer bestimmten Form, Erscheinung, auf bestimmte Art u. Weise existiert u. als solches Gegenstand der Wahrnehmung, Erkenntnis ist“. Diese Definition ist dem Lemma „Ding“ in: Duden - Deutsches Universalwörterbuch. 7. Aufl. Mannheim 2011, entnommen. 
objektiv historische Zeugnisse oder Quellen, sie müssen erst aufgrund unserer Auffassung, unserer neu auftauchenden Fragestellungen oder unserer sich verändernden Sensibilität und teilweise infolge von technologischer Innovation als solche wahrgenommen werden: „Spuren entstehen im Auge des Beobachters“.7 Gewiss gibt es Dinge - etwa die historiografischen Werke, die historischen Berichte oder auch die Denkmäler -, die unmittelbaren Quellencharakter besitzen, weil ihre Urheber sie bewusst aus dem Anliegen heraus schufen, einer bestimmten Persönlichkeit, eines Sachverhalts oder eines Ereignisses zu gedenken und ihre Erinnerung den künftigen Generationen anzuvertrauen. Jedoch können neben diesen Überlieferungen, die sich der Geschichtsschreibung als unmittelbare, absichtlich erzeugte Quellen anbieten, alle Arten von Überresten materieller und immaterieller Natur als Zeugnisse befragt werden.

Besonders anschauliche Beispiele dafür liefern Steinobjekte, die bis in die Frühe Neuzeit als Versteinerungen der vom Donnergott in die Erde geschickten Blitze angesehen wurden und erst ab dem 17. Jahrhundert ${ }^{8}$ von den meisten europäischen Gelehrten als anthropogene Werkzeuge - Faustkeile oder Steinbeile - zunächst vorrömischer und später prähistorischer Zeiten ${ }^{9}$ gedeutet wurden. Erst das Interesse an der lokalen autochthonen vorrömischen Vergangenheit prädisponierte das sonst klassisch geschulte Auge der Gelehrten dafür, diese Gegenstände in den Blick zu nehmen und zu historischen Zeugnissen aufzuwerten. Erwähnt sei ferner, um ein aktuelleres Beispiel anzuführen, das in diesem Sammelband Patrick J. Geary bespricht, die DNA aus menschlichen Knochenresten als Träger genetischer Informationen, mit denen Historiker/-innen aktuell versuchen, ältere Deutungen zur mittelalterlichen Migrationsgeschichte zu entkräften und neue Forschungsfragen zu beantworten. Die Einbeziehung steinerner Dinge in die Sphäre der menschlichen Produktion einer fernen Vergangenheit sowie die Auswertung organischer menschlicher Überreste als materielle Spuren von Wanderungsphänomenen setzt stets eine geistige Operation voraus: zunächst das Herauslösen dieser Dinge aus ihrem vorgefundenen Nutzungs- bzw. Bedeutungskontext und darauffolgend ihre

7 Andreas Buller: Theorie und Geschichte des Spurenbegriffs. Entschlüsselung eines rätselhaften Phänomens. Marburg 2016, S. 72.

8 Zunächst Michele Mercati (1541-1593) in seiner Metallotheca, die jedoch erst 1717 veröffentlicht wurde: Michaelis Mercati Samminiatensis Metallotheca Opus Posthumum [...]. Rom 1717. S. zu dieser Umdeutung: Pierre Saintyves: Pierres magiques, bétyles, haches-amulettes et pierres de foudre. Traditions savantes et traditions populaires. Paris 1936, bes. S. 11-74.

9 Mit den ansprechenden Worten von Marc Bloch gesagt: „Vor Boucher de Perthes konnte man ebenso wie heute eine Menge von Feuersteinen im Schwemmland der Somme finden. Aber es fehlte der Befrager und so gab es keine Urgeschichte“. Ders.: Apologie der Geschichte oder Der Beruf des Historikers. Hrsg. von Friedrich J. Lucas. Stuttgart 1974, S. 75 (frz.: 1949). 
Einbettung in eine neue Sinn- und Quellenkonstellation, deren inneres Zusammenspiel diesen Dingen eine neue Bedeutung verleiht, eben eine historische. Entscheidend für die Einstufung der steinernen Dinge als Zeugnisse prähistorischer Kulturstadien waren ihre Entdeckung im Rahmen von Grabungskontexten sowie ihr Vergleich mit steinernen Artefakten, die in der „Neuen Welt“ in der Frühen Neuzeit als Werkzeug eingesetzt wurden, wovon europäische Reisende in jener Zeit berichtet hatten. ${ }^{10}$ Auf der anderen Seite wird die DNA nicht erst durch die technische Möglichkeit ihrer Entschlüsselung zum historischen Material, sondern durch die bewusste Entscheidung der Historiker/-innen, sie zum historischen Belegmaterial zu erheben und entsprechend zu befragen.

Das gilt im Übrigen auch für die überlieferten Schriftstücke, die nicht absichtlich mit einem spezifischen historischen Sinngehalt versehen wurden, etwa Rechnungsbücher, Katechismen, Verwaltungsakten, statistische Aufzeichnungen, Zollrollen oder Volkslieder, um nur einige Beispiele zu nennen, die zunächst ihrem „ursprünglichen“ Sinnzusammenhang entrissen werden müssen, um dann für ihnen nicht immanente historische Fragen zur Verfügung zu stehen. Und schließlich vermag es auch eine Quelle, die bewusst hergestellt wurde, um die Um- und Nachwelt zu unterrichten und die als Tradition überliefert wurde, über andere, anfangs nicht vorhersehbare Fragen Auskunft zu geben und in anderen Zusammenhängen eine neue Bedeutung hervorzubringen. Jörn Rüsen hat diesen Vorgang der Zuschreibung von historischer Bedeutung auf den Punkt gebracht: „Was Quelle ist, hängt nämlich schlicht und einfach davon ab, was ich wissen will." ${ }^{11}$

Dass potenziell jedes Material ab einem gewissen Alter als Zeuge der Vergangenheit vernommen werden kann, ist - zumindest auf der theoretischen Ebene - der Grundsatz unseres modernen Quellenverständnisses. Diesen hat für die deutsche Geschichtswissenschaft zunächst Johann Martin Chladenius in seinem Werk Allgemeine Geschichtswissenschaft (1752) ${ }^{12}$ formuliert und Johann Gustav Droysen in seinen Vorlesungen zur Heuristik (1857-1882) systematisiert. Zeugnisse sind für Droysen „Schriftsteller, Akten, Monumente, Gesetze, Zustände, Überbleibsel aller Art, von denen wir freilich wissen, daß ihr Ursprung in andere

10 Dies bezeugen z.B. die Beiträge von Antoine de Jussieu zu verschiedenen Gesteinen und Steinbeilen: De l'origine et des usages de la Pierre de Foudre. In: Mémoires de l'Académie Royale des Sciences, année M. DCCXXIII. Paris 1753, S. 6- 9, sowie den Bericht zu seinem Memoire in: Sur les Pierres de Foudre, les Yeux de Serpent, \& les Crapaudines. In: Histoire de l'Académie Royale des Sciences, année M. DCCXXIII. Paris 1753, S. 15-17.

11 Rüsen, Historik, S. 176.

12 Johann Martin Chladenius: Allgemeine Geschichtswissenschaft (1752). Einleitung von Christoph Friedrich u. Vorwort von Reinhart Koselleck. Wien [u. a.] 1985. 
und andere Zeiten hinaufreicht; aber sie liegen uns so gegenwärtig vor, daß wir sie erfassen können, und nur weil sie so noch in der Gegenwart stehen, können wir sie erfassen und u. a. als Material historischer Forschung benutzen“. ${ }^{13}$ Eine solche Quellendefinition wird im Laufe des 19. Jahrhunderts in den Lehrbüchern zu historischen Methoden, zumindest im deutschsprachigen Wissenschaftsraum, immer wieder bestätigt. Auch der Optimismus dahingehend, dass seit der Renaissance die systematische Sammlung von historischen Materialien mit zunehmendem Eifer betrieben worden sei und sich die Arten der als Quellen geltenden Überreste stetig vermehrten, gehört $\mathrm{zu}$ diesem präsentistischen Quellenverständnis. $^{14}$

Daraus ergibt sich, dass selbst die Bestimmung dessen, was als „historisches Material“ zu betrachten ist, ebenso wie die changierenden Erwartungen und Orientierungsbedürfnisse zu den geschichtlichen Fakten zählt. Auch diesen Fakten gilt das Interesse des vorliegenden Sammelbands. In dessen Vordergrund stehen nicht primär die Narrative, Diskurse, Rechtfertigungs- bzw. Legitimierungsargumente, sondern eher die Anerkennung, Selektion und Aufbereitung des historischen Materials, das über das jeweilige Wissen-Wollen und über bestimmte historische Fragen Aufschluss gibt. Angestrebt wird damit zunächst, die Geschichte der Geschichtswissenschaft und -schreibung gegen den Strich zu bürsten und sie aus einer ungewöhnlichen Perspektive neu zu beleuchten: Es geht um die Zusammenstellung von Quellenkorpora.

Um den Blick auf den konstruktiven Charakter des historischen Materials zu schärfen, wird in dem Sammelband folglich das Augenmerk auf jene Vergangenheiten gelenkt, die sich an der Grenze der geschichtlichen Rekonstruierbarkeit befinden und hier unter dem Ausdruck „schriftlose Vergangenheiten“ subsumiert werden. Darunter werden vergangene Kulturen, Völker und Akteure, aber auch Ereignisse und Zustände verstanden, die keine oder nur unzulängliche schriftliche bzw. literarische Zeugnisse hinterlassen haben bzw. von denen dies tradiert ist. Für diese Überlieferungssituation mag es unterschiedliche Gründe geben: dass die Schrift als Tradierungsmittel noch nicht entwickelt war, absichtlich nicht verwendet wurde oder andere Tradierungspraktiken bestanden, dass das schriftlich fixierte Gedächtnis gezielt zerstört wurde oder fatalerweise abhandenkam, dass über bestimmte Vergangenheiten keine Selbstzeugnisse, sondern nur indirekte, fremde, als tendenziös eingestufte Schriftquellen aufbewahrt worden sind, nämlich die Zeugnisse der Sieger, der Kolonisatoren, der Macht-

13 Droysen, Historik, S. 9.

14 Hierzu stellvertretend Ernst Bernheim: Lehrbuch der Historischen Methode und der Geschichtsphilosophie. Bd. 1. 5. u. 6. neubearb. u. verb. Aufl. New York 1960, S. 259. 
haber, der Richter etc., oder auch, dass das historisch Rückblickende stetig neue Felder und Aspekte ins Visier nimmt, für die keine schriftliche Dokumentation vorliegt.

Da sich die Geschichtswissenschaft, trotz ihres weiten Quellenverständnisses, primär als eine auf Schriftlichkeit gründende Wissenschaft versteht, stellen diese schriftlosen Vergangenheiten ein Vakuum, eine Grenze oder ein Hindernis auf dem Weg zur historischen Erkenntnis dar, kurzum: ein Problem und eine methodische Herausforderung zugleich. Wie historisch arbeitende Gelehrte und Wissenschaftler/-innen - von der Frühen Neuzeit bis in unsere Gegenwart - sich mit diesem Vakuum auseinandersetzten bzw. auseinandersetzen, welche Überreste sie als Alternativen zu den Schriftquellen herausfanden und herausfinden und welches Selbstverständnis sowie welche Funktion der Geschichte als Wissen(schaft) sie damit behaupteten oder noch heute vermitteln - dies sind die Fragestellungen, die im Mittelpunkt des Sammelbands stehen.

Bevor auf diese Fragestellung näher eingegangen wird, sollen zunächst, ohne Anspruch auf Vollständigkeit, einige Überlegungen angestellt werden, um den engen Zusammenhang zwischen Schrift und Geschichtswissenschaft, wie er sich in der Neuzeit herausgebildet hat, erneut zu hinterfragen.

\section{Die Geschichtswissenschaft im Spiegel der Schriftlichkeit}

Es steht außer Frage, dass die europäische Geschichtsschreibung seit der Frühen Neuzeit bis in die Gegenwart weit vor allen anderen Zeugnisarten schriftliche Aufzeichnungen bevorzugt. Diese galten und gelten immer noch als die zugänglichsten, an Sinnhaftigkeit reichsten Materialien zur Beantwortung historischer Fragen. Hingegen geht die stärkere Fixierung auf die schriftlichen Aufzeichnungen als exklusive, wissenschaftlich gültige Quellen mit einer bestimmten Phase in der Geschichte der Geschichtsschreibung bzw. -wissenschaft einher, die weniger mit ihrer Verwissenschaftlichung ab dem 17. Jahrhundert als vielmehr mit ihrer Institutionalisierung und Etablierung als Leitdisziplin an den europäischen Universitäten seit der zweiten Hälfte des 18. Jahrhunderts und überwiegend aber seit dem 19. Jahrhundert zusammenhängt. ${ }^{15}$ In diesem Zeitraum geriet die Schrift

15 Hier werden die zwei Phasen zeitlich auseinandergehalten, da die Festlegung der Regeln, wie Geschichte anhand überprüfbarer Urkunden und weiterer empirischer Belege zu verfassen sei, ihrer Etablierung als Disziplin im universitären Kontext deutlich vorausgeht. $\mathrm{Zu}$ wichtigen Etappen dieser Entwicklung hat die Forschung jeweils die Kirchen- und Papstgeschichtsschrei- 
sowohl zum zeitlichen Trennungskriterium zwischen Prähistorie und Geschichte als auch zur räumlichen Zäsur zwischen der Vergangenheit der „Völker ohne Geschichte“ und derjenigen Europas und schließlich zur heuristischen Abgrenzung zwischen der Forschung, die im Universitätskontext betrieben, und der als laienhaft angesehenen Geschichte, die in den bürgerlichen Geschichtsvereinen und -museen gepflegt wurde (s. hierzu den Beitrag von Stefan Jordan in diesem Band).

Die Forschung hat sich lange mit dieser Beschränkung der Geschichtswissenschaft auf das Kriterium Schriftlichkeit und die daraus folgende Exklusion weiterer Felder der Vergangenheit beschäftigt - jüngst aus postkolonialer, globalgeschichtlicher Perspektive. ${ }^{16}$ Die dafür genannten Gründe sind zahlreich: Zunächst, so ein Erklärungsversuch, hing dies mit der Etablierung der Geschichtswissenschaft als akademischer Disziplin zusammen. Dies geschah in Abgrenzung von der Geschichtsproduktion in den als dilettantisch angesehenen lokalen Geschichtsvereinen und kulturhistorischen Museen - etwa dem Germanischen Nationalmuseum in Nürnberg ${ }^{17}$-, die gegenüber dinglichen Quellen und mündlichen Überlieferungen weitaus offener waren als Berufshistoriker. ${ }^{18} \mathrm{Au}$ ßerdem wurde die Ausklammerung schriftloser Kulturen aus der Geschichtswissenschaft mit dem Auftauchen anderer, miteinander konkurrierender wissen-

bung eines Caesar Baronius (1538 - 1607) erklärt, die „Académie“ der französischen Benediktiner - vertreten vor allem von Jean Mabillon (1632-1707) und Bernard de Montfaucon (1655-1741) oder, noch bevor Johann Christoph Gatterer (1727-1799) 1764 die Historische Akademie in Göttingen gründete, Johann Martin Chladenius (1710 - 1759).

16 Henri Moniot: L'histoire des peuples sans histoire. In: Faire de l'histoire. Nouveaux problèmes, nouvelles approches, nouveaux objets. Hrsg. von Jacques Le Goff u. Pierre Nora. Paris 1974, S. 149-171; Eric R. Wolf: Europe and the People without History. Berkeley (Kalifornien) [u. a.] 1982; Christoph Marx: „Völker ohne Schrift und Geschichte“. Zur historischen Erfassung des vorkolonialen Schwarzafrika in der deutschen Forschung des 19. und frühen 20. Jahrhunderts. Stuttgart 1988; Jürgen Osterhammel: „Peoples without History“ in British and German Historical Thought. In: British and German Historiography 1750 - 1950. Hrsg. von Benedikt Stuchtey u. Peter Wende. Oxford 2000, S. 265-287; Andrew Zimmerman: Anthropology and Antihumanism in Imperial Germany. Chicago [u.a.] 2001, bes. S. 38-61; Andreas Eckert: Historiography on a „Continent without History“. Anglophone West Africa, 1880s-1940s. In: Across Cultural Borders. Historiography in Global Perspective. Hrsg. von Eckhardt Fuchs u. Benedikt Stuchtey. Lanham (Maryland) [u.a.] 2002, S. 99-118.

17 Grundlegend hierzu Bernward Deneke u. Rainer Kahsnitz: Das Germanische Nationalmuseum Nürnberg 1852-1977. Beiträge zu seiner Geschichte. München/Berlin 1978; weiterhin erhellend für die vorliegende Fragestellung: Walter Hochreiter: Vom Musentempel zum Lernort. Zur Sozialgeschichte deutscher Museen 1800 -1914. Darmstadt 1994, S. 58-86.

18 Jüngst hierzu Peter N. Miller: History and its Objects. Antiquarianism and Material Culture since 1500. Ithaca (New York) 2017, bes. S. 123-139. 
schaftlicher Paradigmen gerechtfertigt, die aus der „Rippe der Geschichte“ hervorgegangen waren und sich seit dem 19. Jahrhundert zu selbstständigen Disziplinen entwickelten, in primis die Archäologie, die Anthropologie, die Ethnologie und die Volkskunde (s. dazu die Beiträge von Hans Peter Hahn, Gudrun M. König/ Elisabeth Timm und Nathan Schlanger). Eine weitere Ursache könnten die mangelnden Sprachkompetenzen gewesen sein, denn im 19. Jahrhundert waren die älteren Sprachsysteme nur unzureichend entschlüsselt und über Kenntnisse in den nicht-klassischen Sprachen - etwa den slawischen oder arabischen - verfügten Historiker in der Regel nicht, wie Stefan Jordan in dem vorliegenden Beitrag u. a. feststellt. Schließlich seien noch die Positionen von Historiker/innen wie Andrew Zimmerman genannt, die jüngst den Chauvinismus und Imperialismus der deutschen Geschichtswissenschaft im 19. Jahrhundert als weitere Gründe für diese Exklusion angeführt haben. ${ }^{19}$

$\mathrm{Zu}$ diesen Analysen, die sich stark auf das 19. Jahrhundert konzentrieren, möchte ich im Folgenden einige Überlegungen beisteuern, die die Gründe dieser Fixierung auf Schriftlichkeit weiter zurückverfolgen, nämlich bis ins 17. und 18. Jahrhundert. ${ }^{20}$

\subsection{Was das Alte Testament der Geschichte vererbte: ein Vakuum, das Geschichtsschreiber nicht füllen konnten oder wollten}

Eine wichtige Rolle in der Frage nach dem Ausschluss schriftloser Vergangenheiten aus der geschichtlichen Betrachtung spielte die Leerstelle in der menschlichen Ursprungsgeschichte, die durch die zunehmende Infragestellung des vorsintflutlichen Narrativs im Buch Moses entstand und das Thema für „Umbesetzungen“ freigab. Einerseits führte die frühneuzeitliche „Entdeckung“ neuer Erdteile und bisher unbekannter Bevölkerungen zu Fragen nach deren Herkunft und der Einheit des Menschengeschlechts, die das Alte Testament - trotz Hilfestellung des Pseudo-Berossus ${ }^{21}$ - nicht überzeugend zu beantworten wusste. Andererseits stellten nicht-europäische Systeme der Zeitrechnung, etwa die in-

19 Zimmerman, Anthropology, bes. S. 38-61.

20 Die folgenden Überlegungen gehen aus meiner Beschäftigung mit der Konstruktion der „keltischen“ Vergangenheit im Frankreich des 18. und 19. Jahrhunderts im Kontext meines Habilitationsvorhabens hervor.

21 S. das immer noch grundlegende Werk von Giuliano Gliozzi: Adamo e il nuovo mondo. La nascita dell'antropologia come ideologia coloniale: dalle genealogie bibliche alle teorie razziali (1500 - 1700). Mailand 1977, hierzu S. 16 u. 28 f. 
dische und vor allem die chinesische, die die Erschaffung der Welt um einige Millennien früher ansetzten, die aus dem Alten Testament gefolgerte Chronologie der Welt infrage - selbst die in der Septuaginta-Fassung. ${ }^{22}$ Einen weiteren, grundlegenden Faktor bei der Entstehung dieser „Leerstelle“ stellt der historische Pyrrhonismus dar, den Descartes mit seiner Hinterfragung der Gewissheit historischer Erkenntnis und Tradition ausgelöst hatte und der sich in verschiedenen Varianten bis ins 18. Jahrhundert immer wieder erneuerte. ${ }^{23}$ Der durch die Skeptiker erzeugte Druck, empirische Belege für die historische Überlieferung zu erbringen, hatte für die Erforschung und die Erzählung der Menschheitsgeschichte erhebliche Konsequenzen.

Auf der einen Seite gaben historisch arbeitende Gelehrte die Erkundung des heiklen Terrains der Ursprungsgeschichte schlichtweg auf. Paradigmatisch dafür ist die Neuorientierung, die die Benediktiner von Saint-Germain-des-Prés hinsichtlich der historischen Forschung in Frankreich vollzogen: Sie, die einige Historiker/-innen mit guten Argumenten zu den Gründern der modernen Geschichtswissenschaft zählen, ${ }^{24}$ wandten sich seit Ende des 17. Jahrhunderts von den Diskussionen über die ursprüngliche Weltgeschichte ab und widmeten sich zunehmend der eigenen Ordensgeschichte, die sie zeitgleich mit der merowingischen Herrschaft in Frankreich ansetzen ließen, sowie der weltlichen Geschichte der französischen Provinzen. ${ }^{25}$

22 Hierzu das erhellende Kapitel I tempi della storia umana in Paolo Rossi: I segni del tempo. Storia della Terra e storia delle nazioni da Hooke a Vico (1979). Mailand 2003, S. 150 - 225.

23 Carlo Borghero: La certezza e la storia. Cartesianesimo, pirronismo e conoscenza storica. Mailand 1983; Markus Völkel: „Pyrrhonismus historicus“ und „fides historica“. Die Entwicklung der deutschen historischen Methodologie unter dem Gesichtspunkt der historischen Skepsis. Frankfurt a.M. [u. a.] 1987; Richard H. Popkin: The History of Scepticism. From Savonarola to Bayle. Verb. u. erw. Aufl. New York 2003; Carlos Spoerhase (Hrsg.): Unsicheres Wissen. Skeptizismus und Wahrscheinlichkeit 1550 - 1850. Berlin/New York 2009; Anton M. Matytsin: The Specter of Skepticism in the Age of Enlightenment. Baltimore 2016. Zum Skeptizismus gegenüber der römischen Historiografie im 18. Jahrhundert s. das unübertroffene Werk von Mouza Raskolnikoff: Histoire romaine et critique historique dans l'Europe des lumières. La naissance de l'hypercritique dans l'historiographie de la Rome antique. Straßburg 1992.

24 S. vor allem die Studien von Blandine Barret-Kriegel: Les historiens et la monarchie. Bd. 1: Jean Mabillon. Paris 1988; Jean Mabillon: Brèves réflexions sur quelques règles de l'histoire. Mit Vorwort u. Anmerkungen von Blandine Barret-Kriegel. Paris 1990, S. 1-35, sowie die prägnante Zusammenfassung von Manfred Weitlauff: Die Mauriner und ihr historisch kritisches Werk. In: Historische Kritik in der Theologie: Beiträge zu ihrer Geschichte. Hrsg. von Georg Schwaiger. Göttingen 1980, S. 153-209.

25 Maurice Lecomte: Les Bénédictins et l'Histoire des provinces aux XVII ${ }^{\mathrm{e}}$ et XVIII ${ }^{\mathrm{e}}$ siècles. Ligugé (Vienne) 1928. 
Auf der anderen Seite vermehrten sich die Versuche, die Beweisführung zum menschlichen Ursprung mithilfe neuartiger, nicht-schriftlicher Zeugnisse auf eine neue Basis zu stellen. Materielle und immaterielle Überreste der Vergangenheit wurden zunehmend als Belege eingesetzt, wenn die schriftliche Tradition infrage gestellt wurde oder es keine schriftliche Überlieferung gab (s. hierzu den Beitrag von Lisa Regazzoni). In Ermangelung von schriftlichen Dokumenten schienen die vielfältigen und polysemen Überreste - etwa Tierfossilien und versteinerte $\mathrm{Mu}$ scheln, Denkmäler, Münzen, Gemmen und Siegel, Toponyme, Patronyme, Dialekte, Sprichwörter, Fabeln und Lobgesänge, Bräuche, Sitten, Trachten und sogar der Volksaberglaube - etwas Fundamentales zu versprechen: ein wahrhaftiges, authentisches, ursprüngliches und unmittelbares Zeugnis der Vergangenheit zu sein. Als Referenz- und Belegmaterial erfüllten diese Überreste die Funktion, (natur-)historische Aussagen empirisch zu untermauern. Diese Spuren früherer Zeiten spielten eine unentbehrliche Rolle, wenn es um als schriftlos geltende Vergangenheiten oder aber um kulturelle und religiöse Aspekte ging, die in den Schriftstücken nicht erwähnt wurden. Dies lässt sich z.B. in der Frühen Neuzeit bei der historischen Erforschung von Völkern beobachten, die entweder in einiger geografischer Entfernung von den europäischen Ländern angesiedelt waren oder in fernen - biblischen oder vorrömischen - Vergangenheiten der europäischen Geschichte gelebt hatten. Auf der einen Seite führte die Begegnung der Europäer mit den autochthonen, bis dahin unbekannt gebliebenen Kulturen zu einem Übersetzungsproblem zwischen unterschiedlichen Kulturcodes, Modi der Aufzeichnung bzw. Tradierung der Vergangenheit und Vorstellungen von Zeit und Geschichte, die miteinander nicht kompatibel waren (s. hierzu den Beitrag von Antonella Romano). Gleichzeitig regten in Europa Identitätsbedürfnisse diverse historisch arbeitende Gelehrte dazu an, autochthone, nicht- bzw. vorrömische Kulturen vor allem im nordeuropäischen Raum zu untersuchen. Darauffolgend wurden ab dem 17. Jahrhundert als einheimisch gedeutete „ethnografische“, „archäologische“ und „linguistische“ Spezimina miteinander verflochten, um das Schweigen der Geschichte zu brechen (und zwar in einem Zusammenspiel, das, wie die Beiträge von Nicole Immig und Bettina Severin-Barboutie zeigen, noch im 19. respektive im 20. Jahrhundert von nicht professionellen Historiker/-innen praktiziert wurde und wird). Beispielsweise wurden Megalithbauten als Zeugnisse druidischer Kultpraktiken oder aber als Gräber nicht- bzw. vorrömischer Völker, etwa der Kelten, Bretonen oder Germanen, gedeutet. ${ }^{26}$ Götternamen der Irokesen wurden anhand

26 Für die französische Diskussion über die Megalithen im europäischen Kontext verweise ich auf Lisa Regazzoni: Als die „groben Steine“ Keltisch sprachen. Die Megalithen als Quellen altgallischer Geschichte im Frankreich des 18. Jahrhunderts. In: Francia 42 (2015), S. 111-134; dies.: Stoff für die Geschichte. Die Megalithen als Geschichtsdinge im Frankreich der 1720er Jahre. In: 
von morphologischen Vergleichen mit altsprachlichen Spezimina zu Belegen für die Abstammung der nordamerikanischen Völker aus Thrakien. ${ }^{27}$ Volkstümliche Redewendungen in bretonischer Sprache wie „Gebe etwas zur Mistel, das Neujahr ist da“ galten als Überbleibsel der druidischen Verehrung dieser Pflanze vor Ort (s. dazu den Beitrag von Lisa Regazzoni). Muschel- und Fischfossilien, die im alpinen Raum aufgefunden wurden, deuteten Gelehrte im 17. und 18. Jahrhundert zunehmend als Dokumente der historischen Entwicklung der Natur und als Zeugnisse der größeren Ausdehnung der Meere zu uralten Zeiten. ${ }^{28}$

Darüber hinaus diente der Vergleich zwischen den Gebrauchsgegenständen, Fabeln, Sitten und Trachten der räumlich fernen „Wilden“ mit denjenigen der zeitlich fernen „alten Europäer“ dazu, Auskunft über die weit zurückliegende Vergangenheit der europäischen Welt zu geben. Dieser Vergleich beruhte auf der Annahme, dass die „Wilden“ das vorschriftliche, ursprüngliche Stadium der europäischen Kultur widerspiegelten. Die Vorstellung, dass die „europäischen“ Kulturstadien sich letztlich nicht nur nacheinander in eine diachrone Fortschrittslinie einordnen, sondern auch in der synchronen Raumdimension wiederfinden lassen, brachte der Ökonom Anne Robert Jacques Turgot 1750 wirkungsvoll zum Ausdruck: Die unterschiedlichen Kulturniveaus, die gleichzeitig auf der Erdoberfläche bestünden, bezeichnete er als die Monumente sämtlicher Schritte der menschlichen Geistesgeschichte, von denen sie ihm zufolge Zeugnis ablegten. $^{29}$

Diese Praktiken des Vergleichs festigten schließlich die Überzeugung, die „Wilden“ befänden sich noch in der anfänglichen Entwicklungsphase der Steinzeit - eine Vorstellung, die ihnen den Zugang zur Weltgeschichte noch im 19. Jahrhundert verbaute.

Im Zusammenhang mit der Fixierung auf die Schriftlichkeit spielt ein weiterer Aspekt eine wesentliche Rolle: Die Praktiken der Beobachtung und Beweisführung, die die Erforschung menschlicher Geschichte gewissermaßen mit derjenigen der Naturgeschichte teilte, konnten selten Narrative produzieren. Der Er-

Objekte als Quellen der historischen Kulturwissenschaften. Hrsg. von Annette Caroline Cremer u. Martin Mulsow. Köln [u.a.] 2017, S. 225-244.

27 Joseph François Lafitau: Mœurs des sauvages ameriquains, comparées aux mœurs des premiers temps. Bd. 1. Paris 1724, S. 126-135.

28 Zur Umdeutung der Fossilien in der Neuzeit und ihrer Aufwertung als Dokumente einer „historisierten“ Naturgeschichte s. Rossi, I segni del tempo; jüngst hierzu Alessandro Ottaviani: Stanze sul tempo. Sei variazioni tra rovine, fossili e vulcani. Rom 2017.

29 Anne-Robert-Jacques Turgot: Second discours. Sur les progrès successifs de l'esprit humain (1750). In: Euvres. Mit Anmerkungen von Dupont de Nemours. Bd. 4. Paris 1844, S. 597-611, hier S. 599. 
kenntnisgewinn, den einige Gelehrte aus der Untersuchung von nicht-schriftlichen Zeugnissen erzielten, kam anderen im Vergleich zu der an Auskünften reichen literarischen Überlieferung als belanglos und mangelhaft vor. Anhand von materiellen und immateriellen Dingen (in der heutigen Sprache: von archäologischen, ethnografischen und linguistischen Zeugnissen) ließen sich eher Abhandlungen über kulturelle sowie kultische Ausprägungen und Zustände schreiben sowie technische und stilistische Entwicklungen und anhand dieser im besten Fall Wanderungen von Völkern nachvollziehen, kaum aber Kausalketten, Ereignisfolgen, menschliche Beweggründe oder gar Politikgeschichte rekonstruieren. Häufig gelang es durch diese Funde lediglich, historiografische Auffassungen zu berichtigen, zu ergänzen oder infrage zu stellen.

Die Praktiken der Zusammenstellung und des morphologischen Vergleichs empirischer Überreste, die als „antiquarisch“ bezeichnet wurden, ${ }^{30}$ schienen ab dem 18. Jahrhundert die „historische Frage“ nach der schriftlosen, ursprünglichen Vergangenheit nicht befriedigend beantworten zu können. Die empirische Herangehensweise fiel dem Wahrheitsanspruch - wie auch dem beißenden Spott der Geschichtsphilosophie anheim. Zuerst waren es „Geschichtsphilosophen“, etwa Voltaire, ${ }^{31}$ Johann Joachim Winckelmann ${ }^{32}$ und später Friedrich Nietzsche, ${ }^{33}$ aber auch Historiker wie beispielsweise August Ludwig Schlözer ${ }^{34}$ oder der Universalgelehrte John Evans (s. hierzu den Beitrag von Schlanger), die immer wieder die antiquarische Arbeitsweise tadelten. Aus ihrer Sicht konnte dieses empirische Vorgehen nur Gelehrtheit vermehren, kleinteilige und irrelevante Details über die Vergangenheit ans Licht bringen und lediglich zur Akkumulation pedantisch zusammengetragener Informationen führen, die eine „trockene“ Neugierde befriedigen, aber kein wahrhaftiges Wissen über die kausalen Zusammenhänge historischer Geschehnisse generieren. Voltaires Geschichtsphilosophie ordnete

30 Es ist freilich schwierig, diese Praktiken fest mit der Figur des „Antiquars“ zu verbinden, weil dies die Existenz einer klar definierbaren Kategorie von Gelehrten suggeriert, die von derjenigen der Historiker getrennt ist. In der Tat konnte ein und derselbe Gelehrte gleichzeitig als „Antiquar“ oder aber als „Historiker“ bezeichnet werden, je nach Charakter und Forschungsmethode seiner Abhandlung.

31 S. hierzu das Kapitel La singe antiquaire in: Jean Seznec: Essais sur Diderot et l'antiquité. Oxford 1957, S. 79-96.

32 Johann Joachim Winckelmann: Vorrede. In: Ders.: Geschichte der Kunst des Alterthums. Dresden 1764, S. IX-XXVI, bes. S. XVII.

33 Friedrich Nietzsche: Unzeitgemässe Betrachtungen. Zweites Stück:Vom Nutzen und Nachtheil der Historie für das Leben. Leipzig 1874.

34 S. August Ludwig Schlözer: Theorie der Statistik. Göttingen 1804, S. 32f. S. mit Bezug auf die „nordischen Antiquare“ Helmut Zedelmaier: Der Anfang der Geschichte. Studien zur Ursprungsdebatte im 18. Jahrhundert. Hamburg 2003, S. 178. 
folglich die antiquarische, objektbezogene Herangehensweise der aufgeklärten Vernunft unter. Diese hatte es sich zur Aufgabe gestellt, allen glaubwürdigen Autoren und Zeugnissen zum Trotz, sämtliche Fakten einer Prüfung auf rationale Plausibilität zu unterziehen. Die Abwertung des Antiquarianismus ging zumindest im aufklärerischen Frankreich mit der Unterordnung der welthistorischen Erkenntnis unter die vernunftbegründete Beurteilung einher.

Die unbesetzt gebliebenen schriftlosen Vergangenheiten wurden im Rahmen geschichtsphilosophischer Betrachtungen zu Projektionsflächen für Idealbilder, etwa der vorchristliche chinesische Theismus (Voltaire) oder der ursprüngliche, „wilde“ Zustand der Menschen (Rousseau), die als Ideale eine Orientierungsfunktion in der französischen Gesellschaft des 18. Jahrhunderts auszuüben hatten.

Diese Ursprungsnarrative, von denen sich die Geschichtsforschung entweder langsam abwandte oder die durch antiquarische Methoden nicht hervorgebracht werden konnten, wurden zum Proprium der Geschichtsphilosophie. „Mit einer anthropologisch fundierten ,Geschichte der Menschheit““, so Helmut Zedelmaier, „eroberte die Philosophie die von der kritischen Historie preisgegebene Totalität der Geschichte. “35

Im Zuge dieser „Eroberung“ setzte die Philosophie die Ansprüche und Erwartungen fest, die jede Rekonstruktion der Vergangenheit - auch der zeitlich und räumlich fernen Vergangenheit - zu erfüllen hatte: Eine Herausarbeitung der transzendenten, rationalen Kausalität des historischen Fortschritts bzw. Weltgeschehens, die sich nicht damit zufriedengeben konnte, Begebenheiten und Zustände neben- oder nacheinander anzuordnen. Damit etablierte die Philosophie einen Auftrag, mit dem sich an der Schwelle zum 19. Jahrhundert jede Wissenschaft konfrontiert sah, die zur Leitdisziplin der Gesellschaft avancieren wollte. Daher lassen sich die Selbstdarstellung der deutschen Geschichtswissenschaft und die Rechtfertigung ihres Forschungsauftrags in der Gründungszeit besser begreifen, wenn man den Kampf um die Deutungshoheit der Vergangenheit berücksichtigt, den die Geschichtswissenschaft mit der (Geschichts-)Philosophie in der zweiten Hälfte des 18. und verstärkt im 19. Jahrhundert zu führen hatte.

35 Zedelmaier, Der Anfang, S. 10. 


\subsection{Schriftlichkeit als unabdingbare Voraussetzung, um die „Aufgabe des Geschichtsschreibers“ zu erfüllen}

Egal, ob man die Rationalität menschlicher Entwicklung und ihrer Perfektibilität (Voltaire, Condorcet), die Idee eines Aufklärungsprozesses (Schlözer, Kant), den absoluten Geist (Hegel), das höchste sittliche Gute (Wilhelm von Humboldt) oder doch die sittlichen Mächte (Ranke, Droysen) betrachtet: Für keine dieser zukunftweisenden Ideen waren prähistorische oder auch gegenwärtige „wilde“ Kulturen von Belang.

Greift man die Ausgangsfrage erneut auf, was die bedeutendsten Geschichtsschreiber im ausgehenden 18. und in der ersten Hälfte des 19. Jahrhunderts vorwiegend wissen wollten und weshalb, ergibt sich eine recht eindeutige Antwort: Für diese Gelehrten bestand die wahre Aufgabe der Geschichtsschreibung darin, das Wirken „geistiger Mächte“ im historischen Geschehen herauszustellen. Wilhelm von Humboldt zufolge sind die Begebenheiten der Vergangenheit so darzustellen, dass das Streben einer Idee, und zwar des höchsten sittlichen Gutes, „Dasein in der Wirklichkeit“ gewinnt. ${ }^{36}$ Für Leopold von Ranke erschöpft sich die historische Realität ebenso wenig im historischen Geschehen und in der Summe von einzelnen Völkergeschichten. Eine wahre Weltgeschichte war für ihn eine Geschichte, die den geistigen Zusammenhang jeder Einzelheit mit der Gesamtentwicklung erkennt. Was unter geistigem Zusammenhang zu verstehen ist, brachte er deutlich zum Ausdruck: „Die Nationen können in keinem anderen Zusammenhang in Betracht kommen, als inwiefern sie, die eine auf die andere wirkend, nacheinander erscheinen und miteinander eine lebendige Gesamtheit ausmachen“. ${ }^{37}$ Nur die Vergangenheit, die aus seiner Sicht mit diesen geistigen Mächten in Berührung gekommen war oder noch kommen sollte, erlangte für ihn welthistorische Relevanz. Da nichteuropäische Völker - unter ihnen auch das chinesische und das indische Volk - seine Ansicht nach auf diesem Schauplatz keine Rolle spielten, betrachtete Ranke sie als „natürliche“, noch in ihrem Ursprungszustand verharrende Völker, die keine historische Entwicklung durchlaufen hätten. Davon unterscheidet sich aus heutiger Sicht Droysens Verständnis der Geschichte und ihres Gegenstands nur unwesentlich. Auch für ihn bestand die Aufgabe der Geschichte darin, den geistigen Zusammenhang der sittlichen Welt, nämlich „nach ihrem Werden und Wachsen“, zu begreifen. „Die

36 Wilhelm von Humboldt: Über die Aufgabe des Geschichtsschreibers (vorgelesen am 12. April 1821). Leipzig 1946, S. 22.

37 Leopold von Ranke: Vorrede zur Weltgeschichte. In: Ders.: Weltgeschichte. Bd. 1: Von den ältesten historischen Völkergruppen bis zur Emanzipation der germanischen Völker. München 2000, S. 5 f., hier S. 6. 
historische Betrachtung“ - so schreibt Droysen - „fasst die Erscheinungen der sittlichen Welt nach ihrem Gewordensein auf, sie gibt ihnen, die ja nur in dem Hier und Jetzt sind, die Rückschau, in der sie sich selber verstehen lernen; sie zeigt ihnen ihre historische Wahrheit, d. h. die Wahrheit, die in ihrem Gewordensein erkennbar ist und die in ihrem Werden sich hat verwirklichen wollen“. ${ }^{38}$ Während Ranke den Höhepunkt der historischen Entwicklung im europäischen System der Großmächte erkannte, sprach Droysen diesen Status dem preußischen Staat zu, der, so der Gelehrte, mehr als irgendein anderer Staat auf einem Prinzip der Entwicklung als auf einem statischen Grundsatz beruhe. ${ }^{39}$ In Preußen setzte Droysen daher all seine Hoffnungen auf die Realisierung der deutschen Einheit. Trotz dieser Unterschiede glaubten beide Historiker als Protestanten - und in bewusster oder unbewusster Anlehnung an Hegel $^{40}$ - an eine göttliche Weltordnung, in deren unablässiger historischer Entwicklung Europa bzw. Preußen zum Höhepunkt bestimmt sei.

Daraus ergibt sich auch die Fixierung auf die schriftlichen Quellen, aus denen sich den Historikern zufolge diese geistige Entwicklung nachvollziehen lässt. Nach Leopold von Rankes Auffassung beginnt Geschichte erst dort, wo Dokumente verständlich sind und glaubwürdige schriftliche Aufzeichnungen vorliegen. ${ }^{41}$ Die Vorgeschichte sei aus der „Historie“ auszuschließen, weil sie ihrem eigentlichen Prinzip, nämlich der urkundlichen Erforschung, widerspreche. Mit dem Mangel an Schriftquellen begründete Ranke auch den Ausschluss nichteuropäischer Gesellschaften aus der Weltgeschichte. Ihre Erforschung betrachtete er als irrelevant für die Geschichtsschreibung und als Aufgabe der Naturwissenschaften, der Naturgeschichte oder aber der Religionswissenschaft. Etwas komplexer fällt in jenen Jahren die Position Droysens aus, weil er - wohlgemerkt gegen Ranke gerichtet - eine breitere Auffassung dazu vertrat, welches Material für die Arbeit des Historikers adäquat sei, nämlich alles, was von der Vergangenheit als Ergebnis oder Überbleibsel gegenwärtig noch aufzufinden sei. Trotzdem grenzt auch Droysen de facto die Vergangenheit nichteuropäischer Zivilisationen ebenso wie die Urgeschichte aus der Geschichtswissenschaft aus.

38 Droysen, Historik, S. 61.

39 Georg G. Iggers: Deutsche Geschichtswissenschaft. Eine Kritik der traditionellen Geschichtsauffassung von Herder bis zur Gegenwart. Wien [u. a.] 1997, S. 141.

40 Keiner der liberalen Historiker betrachtet sich selbst als Hegelianer. Nichtsdestotrotz: „mehr oder weniger bewusst übernahmen sie jedoch seine Vorstellung vom sittlichen Charakter des Staates und der sinnvollen Entwicklung der Geschichte.“ Iggers, Deutsche Geschichtswissenschaft, S. 126.

41 Ranke, Vorrede, S. 5. 
Aus diesen stichprobenartig angestellten Ausführungen ergibt sich die These, dass die Abwendung der Geschichtswissenschaft einerseits von den zeitlich und räumlich entfernten schriftlosen Vergangenheiten und andererseits von der antiquarischen Arbeit mit mündlichen und sachlichen Quellen auch mit der Veränderung der Erwartungen zusammenhing, die an die historische Darstellung geknüpft wurden. Überspitzt formuliert: Die Geschichtswissenschaft hat im Prozess ihrer Identitätsfindung und Legitimierung als wissenschaftliche und akademische Leitdisziplin gegenüber der Philosophie auf ganze historische Felder und Quellengattungen verzichten wollen und müssen.

\subsection{Grenzorte des Nichtschriftlichen}

Konsequenterweise mündeten die verschiedenen Praktiken der Untersuchung von nichtschriftlichen Überresten im 19. Jahrhundert in die Entstehung neuer Disziplinen, etwa der Archäologie, der Ethnologie, der Anthropologie und der Volkskunde. Diese übernahmen in ihrer Anfangsphase von der Geschichte nicht nur die Methoden, sondern auch die aufgegebenen Forschungsgebiete sowie den Anspruch, historische Erkenntnisse zu gewinnen und historische Erzählungen hervorzubringen (hierzu die Beiträge von Hahn, König/Timm und Schlanger). Ob eine vollständige Emanzipation dieser Disziplinen von der Geschichte möglich war bzw. gelungen ist, bleibt offen, zumal die kulturelle Identität eines „Volkes“ immer noch auf historischen Erzählungen beruht. Umgekehrt blieb das Verhältnis der Geschichtswissenschaft zu diesen Disziplinen zwiespältig. Sie schloss sie einerseits in ihre methodischen Lehrbücher als Teil der Geschichte ein, ${ }^{42}$ indem sie die Nachbardisziplinen zu Hilfswissenschaften erklärte und vorgab, ihre Ergebnisse in die historische Darstellung einzubeziehen. Andererseits profitierte die Geschichte zumindest in der ersten Hälfte des 19. Jahrhunderts kaum von den Ergebnissen anderer Fächer. ${ }^{43}$

Darüber hinaus hatte die Geschichtswissenschaft das Problem der nichtschriftlichen Vergangenheiten mit der Abtretung bestimmter Forschungsgebiete nur vorübergehend aus ihrer disziplinären Welt geschafft. Das Unbehagen gegenüber dieser Einschränkung auf die ausschließliche Heranziehung von

42 Hierzu z.B. Bernheim, Lehrbuch, Bd. 1, S. 85-157, s. insbes. das Verhältnis zwischen Geschichte und Ethnologie (S. 99f.).

43 Paradigmatisch dafür ist die Anerkennung, die Droysen dem Altertumsforscher und Archivar Georg Christian Friedrich Lisch (1801-1883) und seinen Untersuchungen prähistorischer Funde zollte, ohne dass er diese Artefakte oder Geschichtsfelder jemals in seine Arbeit einbezog. S. Droysen, Historik, S. 71f. 
schriftlichen Dokumenten sowie auf die Erforschung der Politik- und Ereignisgeschichte, vor allem in ihrer Ausprägung in der Ranke'schen Schule, kam bei den Historikern seit der zweiten Hälfte des 19. Jahrhunderts zunehmend zum Ausdruck. Beispiele hierfür sind: Numa Denis Fustel de Coulanges' Appell, in Ermangelung von Schriftquellen alle menschlichen Spuren zu befragen; ${ }^{44}$ Camille Jullians Verwertung der archäologischen Überreste und der einfachen Artefakte; ${ }^{45}$ Karl Lamprechts Untersuchungen von Bildmaterial, etwa der ornamentierten Initiale, um eine Geschichte der deutschen Geschmacksentwicklung zu verfassen, ${ }^{46}$ ferner seine gesamten kulturgeschichtlichen Ansätze; die Annales-Schule mit ihrer Abwendung von der Ereignisgeschichte und ihrem stark differenzierten Quellenverständnis, das auch archäologische, kunsthistorische und Schriftstücke zur Erfassung quantitativer Statistiken umfasste; ${ }^{47}$ die griechischen und türkischen Mitglieder von Flüchtlingsvereinen, die auf der Basis von volkstümlichem Material die jeweilige Geschichte der „ethnischen“ Gruppen vor dem Bevölkerungsaustausch von 1923 rekonstruieren wollten (hierzu Immig); der „Gründervater“ der modernen Oral History, Allan Nevins, der mithilfe von mündlichen Berichten, von nicht schriftlich verfassten Erinnerungen, die politischen und kulturellen Aspekte der jüngsten amerikanischen Geschichte vor der Vergessenheit retten wollte; alle darauf folgenden Benutzer dieser Methode, die damit eine Vielstimmigkeit und Demokratisierung der Geschichte im weiten Sinne anstrebten - etwa Lutz Niethammer und seine Befragung der Arbeiter im Ruhrgebiet, um der Masse der Unterschicht eine Stimme zu verleihen und damit eine demokratische

44 Dazu äußerte er sich wie folgt: „La où l'homme a passé, là où il a laissé quelque faible empreinte de sa vie et de son intelligence, là est l'histoire“. Zit. Numa Denis Fustel de Coulanges: Une leçon d'ouverture et quelques fragments inédits de Fustel de Coulanges. In: Revue de synthèse historique 2 (1901), S. 241-263, hier S. 245.

45 Camille Jullian: La Vie et l'Étude des Monuments Français. Leçon d'inauguration de la Chaire d'Histoire et d'Antiquités nationales prononcée au Collège de France le 7 décembre 1905. Paris 1906, S. 10 f.; ders.: Histoire de la Gaule. Bd. 1: Les invasions gauloises et la colonisation grecque. Paris 1920, bes. S. 153-172; ders.: Au seuil de notre histoire. Leçons faites au Collège de France. Bd. 1: 1905-1914. Poitiers 1930, S. 57 f.

46 Karl Lamprecht: Initial-Ornamentik des VIII. bis XIII. Jahrhunderts. Leipzig 1882.

$47 \mathrm{Vgl}$. hierzu die eindrucksvollen Worte Lucien Febvres aus seinem Aufsatz über Marc Blochs posthum erschienenes Werk Apologie de l'histoire ou Métier d'historien: „L'histoire se fait avec des documents écrits, sans doute. Quand il y en a. Mais elle peut se faire, elle doit se faire, sans documents écrits s'il n'en existe point [...] Donc, avec des mots. Des signes. Des paysages et des tuiles. Des formes de champ et de mauvaises herbes. Des éclipses de lune et des colliers d'attelage. Des expertises de pierres par des géologues et des analyses d'épées en métal par des chimistes. D'un mot, avec tout ce qui, étant à l'homme, dépend de l'homme, sert à l'homme, exprime l'homme, signifie la présence, l'activité, les goûts et les façons d'être de l'homme. “ Ders.: Vers une autre histoire (1949). In: Ders.: Combats pour l'histoire. 2. Aufl. Paris 1965, S. 419-438, hier S. 428. 
Geschichte zu entwickeln; all die Historiker/-innen, die weiterhin auf der Baustelle der afrikanischen Geschichte mithilfe von mündlichen und gegenständlichen Zeugnissen arbeiten (hierzu den Beitrag von Nikolas Gestrich); oder aber die Anklage gegen die schriftlichen Dokumente, die zum Ausdruck der Macht werden, die gegen die „anderen“ ausgeübt wird, wie dies Michel de Certeau oder Michel Foucault exemplarisch ausloteten. ${ }^{48}$ Diese sehr knappe Auswahl an Beispielen steht hier stellvertretend für die breit angelegte und stetige Infragestellung einer inhaltlich und methodologisch zu eng gefassten Geschichtswissenschaft. Die genannten Ansätze bestätigen darüber hinaus, dass hinter jeder Zusammenstellung und Aufbereitung von historischen Zeugnissen nicht nur eine historische Frage steht, sondern auch eine Vorstellung dessen, was Geschichte sei und welche Aufgabe sie zu erfüllen habe.

\section{Das Bekenntnis zu den schriftlosen Vergangenheiten und den nicht-schriftlichen Spuren}

Der Rückgriff auf hybride Korpora wird in der gegenwärtigen Forschung unumgänglich, wenn die Erfordernisse der postkolonialen Geschichtsschreibung ernst genommen werden (hierzu den Beitrag von Alessandro Triulzi). Dabei geht es nicht darum, „die Geltungsansprüche fachwissenschaftlicher Erkenntnisleistungen allzu schnell als ideologisch, als obsolet gewordenes westliches Herrschaftsdenken“49 abzutun, sondern darum, zur kritischen Auseinandersetzung mit der modernen Geschichtsschreibung als europäischer Wissensform beizutragen. ${ }^{50}$ Dazu gehört das Bewusstsein, dass die Geschichtsschreibung, wie sie sich ab der Frühen Neuzeit herausgebildet hat, „Europa“ zum souveränen Subjekt aller Geschichten erhoben, die Kategorien des historischen Denkens geprägt und

48 Hierzu Michel de Certeau zu den „Wissenschaften vom „Anderen““ in: Ders.: GlaubensSchwachheit. Hrsg. von Luce Giard. Stuttgart 2009 (frz.: 1987), bes. S. 170 f.; sowie die Summa seiner Auseinandersetzung mit dieser Fragestellung in ders.: Das Schreiben der Geschichte. Frankfurt a.M./New York 1991 (frz.: 1975); zu Michel Foucault s. insbesondere: La vie des hommes infâmes. In: Ders.: Dits et écrits II, 1976-1988. Paris 2001, S. 237-253.

49 Rüsen, Historik, S. 19.

50 S. zu dieser Problemstellung die inzwischen zu Klassikern der Post-colonial Studies gewordenen Beträge von Dipesh Chakrabarty: A Small History of Subaltern Studies. In: Habitations of Modernity. Essays in the Wake of Subaltern Studies. Hrsg. von Dipesh Chakrabarty. Chicago 2002, S. 3-19; ders.: Provincializing Europe: Postcolonial Thought and Historical Difference. Princeton (New Jersey) 2000. 
die explanantes der geschichtlichen Entwicklung für die gesamte Weltgeschichte festgelegt hat, was Historiker/innen aus den einst europäischen Kolonien bis heute Probleme bereitet. ${ }^{51}$ In ihrem Anspruch, Selbsterkenntnis zu üben, wie Heraklit es mit dem Spruch „erkenne dich selbst“52 forderte und worauf sich Droysen unermüdlich berief, hat die historische Erkenntnis unter dem Begriff „Menschheit“ vorwiegend die Europäer/innen erkundet. Dass die europäische Geschichtsschreibung in hohem Maße selbstreferenziell ist, weil sie weitgehend auf selbst produzierten Schriftquellen beruht, ${ }^{53}$ brachte Michel de Certeau in den 1970er-Jahren mit großer Schärfe in seiner Analyse zum Ausdruck, die keineswegs an Aktualität verloren hat (s. dazu auch den Beitrag von Romano). Ebenso zeitgemäß wirkt seine Aufforderung: „Man muss schon neue Verfahren erfinden, die in einer Geschichtswissenschaft anderen Typs auch Erfahrungen ohne Schrift samt der ihnen eigenen Optik ihren Platz finden lassen. “54

All diese Reflexionen haben die Frage, was wir als Historiker/innen über die Vergangenheit wissen wollen, stark verändert. Entsprechend fällt der sich daraus entwickelnde „demokratisierte“ oder zumindest „pluralisierte“ Geschichtsblick vermehrt auf medial ausdifferenzierte Überreste, die andere Praktiken und Methoden der Quellenarbeit verlangen und vor allem multidisziplinäre Fachkompetenzen oder aber Kooperationen erfordern. Das wirkt sich wiederum stark auf das Selbstverständnis der Disziplin „Geschichtswissenschaft“ aus. Um diese Bereiche im Sinne der geltenden wissenschaftlichen Ansprüche, aber in Ermangelung von Schriftquellen zu untersuchen, müssen die Historiker/-innen ,jede Spur als ein Dokument“"55 betrachten. Mauer- und Stadtüberreste, Hügelgräber, Ruinen

51 S. den Überblick in Eckhardt Fuchs: Introduction. Provincializing Europe. Historiography as a Transcultural Concept. In: Fuchs/Stuchtey, Across Cultural Borders, S. 1-28. Vgl. ferner Jack Goody, der nicht nur beklagt, dass Europa dem Rest der Welt seine historischen Begriffe und Periodisierung auferlegt habe, sondern auch, dass Europa bestimmte Lebensformen (etwa die Demokratie, die Freiheit, die Familie) unrechtsmäßig als eigene exklusive Erfindung beansprucht. S. ders.: The Theft of History. Cambridge 2006.

52 Droysen, Historik, S. 28.

53 Michel de Certeau: L'opération historique. In: Le Goff/Nora (Hrsg.), Faire de l'histoire, S. $17-66$, bes. S. $39 \mathrm{f}$.

54 Certeau, GlaubensSchwachheit, S. 171.

55 In Bezug auf die Erforschung der vorkolonialen subsaharischen Geschichte schreibt FrançoisXavier Fauvelle: „La rareté de nos sources crée une obligation, qui est sans aucun doute l'une des caractéristiques les plus fortes du métier d'historien de l'Afrique: celle de considérer chaque trace à l'égal d'un document“: Ders.: Le rhinocéros d'or. Histoire du Moyen Âge africain. Paris 2013, S. 18. Einen guten Überblick über Quellen und Methoden zur Erforschung des „Vorkolonialen“ Afrikas bietet Adam Jones: Afrika bis 1850. Frankfurt a.M. 2016, S. 32-39. S. des Weiteren die ihm gewidmete Festschrift: Geert Castryck [u.a.]: Sources and Methods for African History and Culture. Essays in Honour of Adam Jones. Leipzig 2016. 
eines Salzbergwerks, ein Fund von Goldmünzen, eine Landkarte, landschaftliche Veränderungen sowie einige spärliche arabische Quellen, die indirekt über Handelswege und -orte berichten, all das sind die historischen Materialien, mit denen beispielsweise der Historiker und Archäologe François-Xavier Fauvelle versucht, einige wenige Mosaiksteine der Geschichte Afrikas südlich der Sahara vom 8. bis 15. Jahrhundert zu rekonstruieren. ${ }^{56}$ Selbst die mündliche Überlieferung, die heutzutage Afrikahistoriker/-innen sehr bedingt einsetzen, kann in einer multidisziplinären Rekonstruktion historischer vorkolonialer Kontexte erneut an Zeugniswert gewinnen ${ }^{57}$ (s. den Beitrag von Gestrich). Damit kommen diese Historiker/-innen der Aufforderung de Certeaus nach, dem Gesichtspunkt der „anderen“ gerecht zu werden. Infolge dieser Arbeiten kommen schließlich Wandlungen und Begebenheiten in der afrikanischen Vergangenheit ans Licht, die Historiker/-innen ihr bis in die erste Hälfte des 20. Jahrhunderts im Namen der postulierten Beständigkeit und Immobilität „primitiver“ Gesellschaften abgesprochen hatten. ${ }^{58}$ Die daraus resultierenden Ergebnisse stellen eine Herausforderung auch hinsichtlich der Darstellungsform dar, die das Kriterium des immanenten Zusammenhangs nicht befriedigen kann - ein weiteres schwer zu lösendes Grundproblem der Geschichtsmethodik und -theorie. Lücken und Widersprüche werden konsequent offengelegt. Es entstehen keine einheitlichen (Master-)Erzählungen, sondern fragmentarische Rekonstruktionsansätze, deren narrative Logik sich an den aufgefundenen Spuren entlang herausbildet.

Die Frage, wie mit nicht schriftlich überlieferten Vergangenheiten umzugehen sei, scheint auch in Bezug auf die Vergangenheit von schriftbasierten Gesellschaften aktueller denn je geworden zu sein. Diesen Eindruck bestätigen die Ansätze, die sich in den letzten Jahrzehnten akademisch etabliert haben, wie die Oral History, die all die historischen „subalternen“ Subjekte zur Sprache kommen lässt, die bis dahin von der auf schriftlichen Belegen basierenden Geschichte vernachlässigt oder nur einseitig erfasst wurden; ebenso die jüngst entstandene Genetic History (hier durch den Beitrag von Geary vertreten), deren programmatische Herausforderung darin besteht, unzulänglich schriftlich dokumentierte Phänomene der Wanderung von Menschengruppen, die eine starke Auswirkung auf die damalige politische Ordnung und kulturelle Identität hatten, anhand genetischer Spuren zu rekonstruieren. Auch die rasante Aufeinanderfolge von

56 Fauvelle, Le rhinocéros d'or.

57 Das Problem wird vor allem von den Wissenschaftler/-innen thematisiert, die sich, und zwar vor Ort, mit der vorkolonialen Geschichte Afrikas beschäftigen. Hierzu Théodore Nicoué Gayibor: Sources orales \& histoire africaine. Approches méthodologiques. Paris 2011, bes. S. 195-203. 58 Hierzu sowie zur schwierigen Verortung der Geschichte Afrikas im akademischen Wissenssystem Jones, Afrika, S. $17 \mathrm{f}$. 
historiografischen Neuansätzen, etwa des material, visual oder acoustic turn (hierzu die Beiträge von Jens Jäger und Muriel Favre), ${ }^{59}$ drückt unter anderem das Bedürfnis aus, auf neuartige hybride Quellenkorpora zurückzugreifen, um Fragen der Mentalitäts-, Ideen- oder Wissensgeschichte, der Alltagsgeschichte, der Technik- und Wissenschaftsgeschichte, der Wahrnehmungs- und Emotionsgeschichte, des vergangenen impliziten Wissens, der Gender- oder Migrationsgeschichte (hierzu der Beitrag von Triulzi) etc. zu behandeln. Teilweise ist die politische Brisanz bestimmter historischer Fragen derart hoch, dass der Einsatz neuartiger Spuren und mühseliger Forschungsmethoden unerlässlich wird, um Mastererzählungen zu dekonstruieren, die konfliktgeladene, ethnisch-identitäre Ansprüche untermauern - etwa der DNA-Untersuchung in der mittelalterlichen Migrationsgeschichte (Geary) oder der Einbeziehung von Flora und Fauna aus vorkolonialer Zeit in der Erforschung der Geschichte der französischen Insel La Réunion (Severin-Barboutie).

Die Vervielfachung der Subdisziplinen und Quellenmethoden ist ein wichtiger Indikator dafür, dass die Geschichtswissenschaft als Disziplin weitgehend auf einer Heuristik beruht, die sich de facto immer weniger exklusiv über die Schrift und ihre Zeugnisse definieren lässt, vor allem, indem sie sich zu einem globalen, differenzierten, polysemen Blick auf die Vergangenheit bekennt.

Der wichtigste Gewinn aus all diesen Ansätzen besteht darin, dass sich diese nicht darauf beschränken, Lücken im historischen Wissen zu schließen bzw. dem erreichten Wissensstand zusätzliche Informationen hinzuzufügen. Vielmehr erschließen sie zugleich neue Forschungsfelder und generieren neue Fragen; sie veranlassen multidisziplinäre Herangehensweisen, die zum stetigen Neu-Arrangement der Disziplinen im Wissenschaftssystem beitragen; sie eröffnen bis jetzt vernachlässigten, ausgegrenzten Individuen und Menschengruppen, Sachverhalten und Sichtweisen Eingang in die Geschichte; sie brechen mit vereinheitlichenden mythisierenden Meistererzählungen und lassen die Multivokalität historischer Realitäten zu.

59 Hier werden nur die turns erwähnt, die im vorliegenden Band behandelt werden, stellvertretend für die zahlreichen weiteren Ansätze, die zur Zeit bestehen. Zur Orientierung im Wald der turns s. Doris Bachmann-Medick: Cultural Turns. Neuorientierungen in den Kulturwissenschaften. Reinbek bei Hamburg 2006. 


\section{Plädoyer für eine Geschichtswissenschaft bis zu den Grenzen und darüber hinaus}

Zur Untersuchung des Umgangs mit schriftlosen Vergangenheiten ist ein knapper Fragenkatalog verfasst worden, der den Autor/-innen dieses Sammelbands zur Orientierung vorlag und sich in vier Fragenkomplexe gliedert. Der erste betrifft die Verfasser einer bestimmten Geschichte und ihre Standortbestimmung, ihren Bezug zu der erforschten Vergangenheit, ob er überhaupt bestehe oder ob er nur imaginiert worden sei, die Gründe, Identitäts- und Orientierungsbedürfnisse, die religiösen oder politischen Ansprüche, die sie bewegen, sowie die Kriterien, anhand derer sie Autorität in dem Forschungsgebiet beanspruchen (bspw. als Zeuge, Nachfahre, Eingeborener oder aufgrund besonderer wissenschaftlicher Expertise). Der zweite Komplex betrifft Fragen nach der Epistemologie der historischen Quellen: wie Historiker/-innen den Mangel an Schriftzeugnissen erklären, ob es sich um eine Vergangenheit handele, in der man keine Schrift gekannt bzw. verwendet habe, oder ob die Schriftzeugnisse (un-)absichtlich getilgt worden seien und weshalb, welche künstlichen, organischen oder immateriellen Überreste im Laufe der Zeit zum Status historischer Quellen erhoben worden seien, um neues Belegmaterial bereitzustellen, welche Denkmuster und hermeneutischen Mittel die Auswertung dieser Quellen unterstützen bzw. verhindern, anhand welcher disziplinären (neuen) Methoden die herangezogenen „Überreste“ zum Sprechen gebracht worden seien und welche Erwartungen auf Antworten sie erweckt hätten. Ein drittes Fragenbündel fokussiert sich auf die historische Erzählung und die Darstellungsform, nämlich, wie die zu untersuchende Vergangenheit räumlich und zeitlich eingegrenzt worden sei, welches Vorwissen über das Forschungsfeld und welche neuen historischen Erkenntnisse aus nicht-schriftlichen Quellen gewonnen worden seien, inwiefern sie herkömmliche Narrative berichtigten, ergänzten oder sogar widerlegten, welche Aussagen damit ausgeschlossen würden und welche Darstellungsform sich für die Präsentation der erzielten Erkenntnisse eigne. Das vierte Fragenbündel dreht sich um die Definition und Standortbestimmung der Geschichte im Spannungsfeld des Wissenssystems: Welches Verständnis von Geschichte als Wissensfeld bzw. Disziplin implizit oder explizit vermittelt werde, gegen welches etablierte Muster der Geschichtswissenschaft und gegen welche Methoden sich dieses ggf. durchsetzen müsse, ob all dies das Überdenken von Geschichte und damit Irritationen und Auseinandersetzungen in der Zunft ausgelöst habe, wie sich die Geschichte bzw. die historische Forschung von anderen Disziplinen abgrenze und in welchem Verhältnis sie zu den Hilfswissenschaften stehe, ob sie mit diesen interdisziplinär zusammenarbeite oder lediglich deren Ergebnisse benutze. 
Zur Auseinandersetzung mit diesen Fragen sind Historiker/-innen und historisch arbeitende Wissenschaftler/-innen aus benachbarten Disziplinen eingeladen worden, die aus drei verschiedenen Perspektiven zum vorliegenden Sammelband beigetragen haben: aus einer historiografischen Perspektive, wobei sie sich mit Fallbeispielen von vergangenen und gegenwärtigen Weisen des Herangehens an schriftlose Vergangenheiten auseinandergesetzt haben (Romano, Regazzoni, Immig, Severin-Barboutie); aus einer wissenschaftshistorischen Perspektive, die das zentrale Moment der Ausdifferenzierung der Disziplinen bzw. ihrer Entstehung aus der Rippe der Geschichte im 19. und frühen 20. Jahrhundert in den Blick nimmt (Jordan, Schlanger, König/Timm, Hahn), und aus einer praktischen Perspektive von historisch arbeitenden Wissenschaftlern/-innen - und, im Fall von Giacomo Sferlazzo, eines Künstlers -, die selbst mit hybriden Zeugnissen und mithilfe von interdisziplinären Ansätzen operieren (Geary, Gestrich, Jäger, Favre, Triulzi, Sferlazzo) und von ihrer Herangehensweise an bestimmte schriftlose Vergangenheiten berichten. Diese drei Perspektiven bestimmen auch den dreiteiligen Aufbau des Sammelbandes.

Alle diese Beiträge vermögen zu den gestellten Fragenkomplexen exemplarisch Wichtiges beizusteuern und werfen wiederum neue Fragen auf, die die Identität des Arbeitsgebiets Geschichtswissenschaft in historischer und gegenwärtiger Perspektive zur Debatte stellen: Wie weit kann sich ihr Forschungsterrain ausdehnen, damit sie mit ihrem methodischen Instrumentarium noch Anspruch auf Wissenschaftlichkeit erheben kann? In welchem Bereich muss sie ihre Methodik aufgeben und sich Ansätze anderer Disziplinen aneignen, um ihr WissenWollen zu befriedigen? Dienen die erzielten Auskünfte noch dazu, die historische Erkenntnis zu erweitern, oder sind sie dafür unbrauchbar bzw. irrelevant? Welche Kontinuitäten und Brüche lassen sich bei den Herangehensweisen an nichtschriftliche Zeugnisse aufzeichnen? Wie viele Kompromisse kann und will die Geschichtswissenschaft in der interdisziplinären Arbeitsweise eingehen, damit ihr Selbstverständnis als Disziplin bestehen bleibt? Oder anders formuliert: Wie viel „Fremdes“ - an Methoden, Zeugnisarten, Konzepten - kann sie sich einverleiben und sich dabei immer noch als Geschichtswissenschaft definieren? An ihrer Grenze verdichten sich also die Fragen.

Dieser Sammelband versteht sich als Plädoyer für Grenzüberschreitungen und -erweiterungen, damit die Geschichtswissenschaft in Anbetracht des Pluralismus an Fragen, die die Gegenwart an die Vergangenheit richtet, ein entscheidendes Wort mitreden kann.

Abschließend seien hier noch kurz Droysens Worte in Erinnerung gerufen, die wie ein künftigen Historikern/-innen hinterlassenes Vermächtnis klingt: „Und es ist noch unermesslich viel da, das nur noch nicht gesehen ist, nur darauf wartet, beachtet, richtig gefragt, als noch gegenwärtiger Zeuge von Vergangenem ver- 
nommen zu werden. ${ }^{\text {“60 }}$ Es sind Worte, die einerseits als Aussage, es gebe noch viel zu tun, und damit als Aufforderung zur Arbeit gedeutet werden können, und andererseits als Aufmunterung: Für Historiker und Historikerinnen bleibt noch viel Material übrig.

\section{Literatur}

Bachmann-Medick, Doris: Cultural Turns. Neuorientierungen in den Kulturwissenschaften. Reinbek bei Hamburg 2006.

Barret-Kriegel, Blandine: Les historiens et la monarchie. Bd. 1: Jean Mabillon. Paris 1988.

Bernheim, Ernst: Lehrbuch der Historischen Methode und der Geschichtsphilosophie. 5. u. 6. neubearb. u. verm. Aufl. New York 1960.

Bloch, Marc: Apologie der Geschichte oder Der Beruf des Historikers. Hrsg. von Friedrich J. Lucas. Stuttgart 1974 (frz.: 1949).

Borghero, Carlo: La certezza e la storia. Cartesianesimo, pirronismo e conoscenza storica. Mailand 1983.

Blumenberg, Hans: Die Lesbarkeit der Welt (1981). 5. Aufl. Frankfurt a.M. 2000.

Buller, Andreas: Theorie und Geschichte des Spurenbegriffs. Entschlüsselung eines rätselhaften Phänomens. Marburg 2016.

Castryck, Geert [u.a.]: Sources and Methods for African History and Culture. Essays in Honour of Adam Jones. Leipzig 2016.

Certeau, Michel de: L'opération historique. In: Faire de l'histoire. Nouveaux problèmes, nouvelles approches, nouveaux objets. Hrsg. von Jacques Le Goff u. Pierre Nora. Paris 1974, S. $17-66$.

Ders.: Das Schreiben der Geschichte. Frankfurt a.M./New York 1991 (frz.: 1975).

Ders.: GlaubensSchwachheit. Hrsg. von Luce Giard. Stuttgart 2009 (frz.: 1987).

Chakrabarty, Dipesh: A Small History of Subaltern Studies. In: Habitations of Modernity. Essays in the Wake of Subaltern Studies. Hrsg. von Dipesh Chakrabarty. Chicago 2002, S. 3-19.

Ders.: Provincializing Europe. Postcolonial Thought and Historical Difference. Princeton (New Jersey) 2000.

Chladenius, Johann Martin: Allgemeine Geschichtswissenschaft (1752). Einleitung von Christoph Friedrich u. Vorwort von Reinhart Koselleck. Wien [u. a.] 1985.

Deneke, Bernward u. Rainer Kahsnitz: Das Germanische Nationalmuseum Nürnberg 1852 -1977. Beiträge zu seiner Geschichte. München/Berlin 1978.

Droysen, Johann Gustav: Historik. Hrsg. von Peter Leyh. Stuttgart-Bad Cannstatt 1977.

Eckert, Andreas: Historiography on a „Continent without History“. Anglophone West Africa, 1880s-1940s. In: Across Cultural Borders. Historiography in Global Perspective. Hrsg. von Eckhardt Fuchs u. Benedikt Stuchtey. Lanham (Maryland) [u. a.] 2002, S. 99-118.

Fauvelle, François-Xavier: Le rhinocéros d’or. Histoire du Moyen Âge africain. Paris 2013.

60 Droysen, Historik, S. 68. 
Febvre, Lucien: Vers une autre histoire (1949). In: Ders.: Combats pour l'histoire. 2. Aufl. Paris 1965, S. $419-438$.

Foucault, Michel: La vie des hommes infâmes. In: Ders.: Dits et écrits II, 1976-1988. Paris 2001, S. 237-253.

Fuchs, Eckhardt: Introduction. Provincializing Europe. Historiography as a Transcultural Concept. In: Across Cultural Borders. Historiography in Global Perspective. Hrsg. von Eckhardt Fuchs u. Benedikt Stuchtey. Lanham (Maryland) [u. a.] 2002, S. 1-28.

Fustel de Coulanges, Numa Denis: Une leçon d'ouverture et quelques fragments inédits de Fustel de Coulanges. In: Revue de synthèse historique 2 (1901), S. 241-263.

Gayibor, Théodore Nicoué: Sources orales \& histoire africaine. Approches méthodologiques. Paris 2011.

Gliozzi, Giuliano: Adamo e il nuovo mondo. La nascita dell'antropologia come ideologia coloniale. Dalle genealogie bibliche alle teorie razziali (1500-1700). Mailand 1977.

Hochreiter, Walter: Vom Musentempel zum Lernort. Zur Sozialgeschichte deutscher Museen 1800-1914. Darmstadt 1994.

Humboldt, Wilhelm von: Über die Aufgabe des Geschichtsschreibers (vorgelesen am 12. April 1821). Leipzig 1946.

Iggers, Georg G.: Deutsche Geschichtswissenschaft. Eine Kritik der traditionellen Geschichtsauffassung von Herder bis zur Gegenwart. Wien [u. a.] 1997.

Jones, Adam: Afrika bis 1850. Frankfurt a.M. 2016.

Jullian, Camille: La Vie et l'Étude des Monuments Français. Leçon d'inauguration de la Chaire d'Histoire et d'Antiquités nationales prononcée au Collège de France le 7 décembre 1905. Paris 1906.

Ders.: Histoire de la Gaule. Bd. 1: Les invasions gauloises et la colonisation grecque. Paris 1920.

Ders.: Au seuil de notre histoire. Leçons faites au Collège de France. Bd. 1: 1905-1914. Poitiers 1930.

Jussieu, Antoine de: De l'origine et des usages de la Pierre de Foudre. In: Mémoires de l'Académie Royale des Sciences, année M. DCCXXIII. Paris 1753, S. 6-9.

Ders: Sur les Pierres de Foudre, les Yeux de Serpent, \& les Crapaudines. In: Histoire de l'Académie Royale des Sciences, année M. DCCXXIII. Paris 1753, S. 15-17.

Koselleck, Reinhart: Standortbindung und Zeitlichkeit. Ein Beitrag zur historiographischen Erschließung der geschichtlichen Welt. In: Objektivität und Parteilichkeit in der Geschichtswissenschaft. Hrsg. von Reinhart Koselleck [u. a.]. München 1977, S. 17-46.

Lafitau, Joseph François: Mœurs des sauvages ameriquains, comparées aux mœurs des premiers temps. Paris 1724.

Lamprecht, Karl: Initial-Ornamentik des VIII. bis XIII. Jahrhunderts. Leipzig 1882.

Lecomte, Maurice: Les Bénédictins et l'histoire des provinces aux XVII et XVIII siècles. Ligugé 1928.

Mabillon, Jean: Brèves réflexions sur quelques règles de l'histoire. Vorwort u. Anmerkungen von Blandine Barret-Kriegel. Paris 1990.

Marker, Chris: Les statues meurent aussi. In: Ders.: Commentaires. Bd. 1. Paris 1961, S. 7-23.

Marx, Christoph: „Völker ohne Schrift und Geschichte“. Zur historischen Erfassung des vorkolonialen Schwarzafrika in der deutschen Forschung des 19. und frühen 20. Jahrhunderts. Stuttgart 1988.

Matytsin, Anton M.: The Specter of Skepticism in the Age of Enlightenment. Baltimore 2016. 
Mercati, Michele: Michaelis Mercati Samminiatensis Metallotheca Opus Posthumum [...]. Rom 1717.

Miller, Peter N.: History and its Objects. Antiquarianism and Material Culture since 1500. Ithaca (New York) 2017.

Moniot, Henri: L'histoire des peuples sans histoire. In: Faire de l'histoire. Nouveaux problèmes, nouvelles approches, nouveaux objets. Hrsg. von Jacques Le Goff u. Pierre Nora. Paris 1974, S. 149-171.

Nietzsche, Friedrich: Unzeitgemässe Betrachtungen. Zweites Stück: Vom Nutzen und Nachtheil der Historie für das Leben. Leipzig 1874.

o. V.: Ding. In: Duden - Deutsches Universalwörterbuch. 7. Aufl. Mannheim 2011.

Osterhammel, Jürgen: „Peoples without History“ in British and German Historical Thought. In: British and German Historiography 1750-1950. Hrsg. von Benedikt Stuchtey u. Peter Wende. Oxford 2000, S. 265-287.

Ottaviani, Alessandro: Stanze sul tempo. Sei variazioni tra rovine, fossili e vulcani. Rom 2017.

Popkin, Richard H.: The History of Scepticism. From Savonarola to Bayle. Verb. u. erw. Aufl. New York 2003.

Ranke, Leopold von: Weltgeschichte. Bd. 1: Von den ältesten historischen Völkergruppen bis zur Emanzipation der germanischen Völker. München 2000.

Raskolnikoff, Mouza: Histoire romaine et critique historique dans l'Europe des lumières. La naissance de l'hypercritique dans l'historiographie de la Rome antique. Straßburg 1992.

Regazzoni, Lisa: Als die „groben Steine“ Keltisch sprachen. Die Megalithen als Quellen altgallischer Geschichte im Frankreich des 18. Jahrhunderts. In: Francia 42 (2015), S. $111-134$.

Dies.: Stoff für die Geschichte. Die Megalithen als Geschichtsdinge im Frankreich der 1720er Jahre. In: Objekte als Quellen der historischen Kulturwissenschaften. Hrsg. von Annette Caroline Cremer u. Martin Mulsow. Köln [u. a.] 2017, S. 225-244.

Rossi, Paolo: I segni del tempo. Storia della Terra e storia delle nazioni da Hooke a Vico (1979). Mailand 2003.

Rüsen, Jörn: Historik. Theorie der Geschichtswissenschaft. Köln [u. a.] 2013.

Saintyves, Pierre: Pierres magiques, bétyles, haches-amulettes et pierres de foudre. Traditions savantes et traditions populaires. Paris 1936.

Schlözer, August Ludwig: Theorie der Statistik. Göttingen 1804.

Seznec, Jean: Essais sur Diderot et l'antiquité. Oxford 1957.

Spoerhase, Carlos (Hrsg.): Unsicheres Wissen. Skeptizismus und Wahrscheinlichkeit 1550-1850. Berlin/New York 2009.

Turgot, Anne-Robert-Jacques: Second discours. Sur les progrès successifs de l'esprit humain (1750). In: Euvres. Mit Anmerkungen von Dupont de Nemours. Bd. 4. Paris 1844, S. $597-611$.

Völkel, Markus: „Pyrrhonismus historicus“ und „fides historica“. Die Entwicklung der deutschen historischen Methodologie unter dem Gesichtspunkt der historischen Skepsis. Frankfurt a.M. [u.a.] 1987.

Weitlauff, Manfred: Die Mauriner und ihr historisch kritisches Werk. In: Historische Kritik in der Theologie. Beiträge zu ihrer Geschichte. Hrsg. von Georg Schwaiger. Göttingen 1980, S. $153-209$.

Winckelmann, Johann Joachim: Geschichte der Kunst des Alterthums. Dresden 1764.

Wolf, Eric R.: Europe and the People without History. Berkeley (Kalifornien) [u. a.] 1982. 
Zedelmaier, Helmut: Der Anfang der Geschichte. Studien zur Ursprungsdebatte im 18. Jahrhundert. Hamburg 2003.

Zimmerman, Andrew: Anthropology and Antihumanism in Imperial Germany. Chicago [u.a.] 2001. 
